\title{
SIGNAL ADAPTIVE POSTPROCESSING FOR BLOCKING EFFECTS REDUCTION IN JPEG IMAGE
}

\author{
H. C. Kim* and H. W. Park \\ Dept. Informatiom and Communication Eng., KAIST \\ 207-43 Cheongryangni, Dongdaemungu \\ Seoul 130-012, Korea \\ hwpark@sicom.kaist.ac.kr \\ *LG Information \& Communication, Ltd.
}

\begin{abstract}
A postprocessing algorithm is proposed to reduce the blocking artifacts of joint photographic experts group (JPEG) decompressed images. The reconstructed images from JPEG compression produce noticeable image degradation near the block boundaries, in particular, for highly compressed images because each block is transformed and quantized independently. The reduction of these blocking effects has been an essential issue for high quality visual communications. The proposed postprocessing algorithm reduces these blocking artifacts efficiently. A comparison study between the proposed algorithm and other postprocessing algorithms is made by computer simulation with several JPEG images. These simulation results show that the proposed algorithm reduces the blocking artifacts significantly in the subjective and objective views.
\end{abstract}

\section{INTRODUCTION}

JPEG had been recommended as a standard compression scheme for continuous-tone still images [1]. JPEG uses an $8 \times 8$ pixel-block discrete cosine transform (DCT) for information packing into a few transform coefficients. This block DCT scheme takes advantage of the local spatial correlation property of images and also saves processing time [2]. However, it is well known that this individual processing of each block induces visually annoying blocking effects [3][4][5], in particular, when a large quantization parameter is used for high compression.

There are three kinds of blocking effects in JPEG decompressed images. One is the staircase noise along the image edges, another is the grid noise in the monotone area, and the other is the corner outlier in the corner point of the $8 \times 8$ DCT block. When an $8 \times 8$ block includes an image edge, the edge is degraded such that the block boundary looks like the edge. This artifact is called staircase noise. A slight change of image intensity between the $8 \times 8$ block boundaries is easily noticeable in the monotone area. This change is called grid noise. A corner outlier is visible at the corner point of the $8 \times 8$ block, where the corner point is either much larger or much smaller than the neighboring pixels.

Several postprocessing algorithms have been proposed to reduce the blocking effects of block-based coding [3][4]. A linear space-invariant low-pass filtering can reduce the blocking effects, but it also degrades image details such as edge and texture, and reduces the image contrast. In order to overcome this degradation problem, a signal adaptive filter can be applied to the JPEG decompressed image. In the signal adaptive filter, the filter frequency response should be varied in accordance with the local signal and noise characteristics. In the Ramamurthi algorithm[3], the ratio of the intensity change is compared to the average intensity with a threshold.

In this paper, a new postprocessing algorithm based on a signal adaptive filtering and on a corner outlier detection/replacement scheme is proposed. In order to preserve the image details, a local threshold value is defined by using the local variance, mean, and global threshold of the gradient image [6][7][8][9]. The staircase noise is smoothed by a 1-D directional filter according to the directions of the edge. The grid noise is smoothed by a signal adaptive 2-D low-pass filtering whose kernel size is $5 \times 5$. The corner outlier [10][11] detection/replacement scheme consists of two stages. At the first stage, the corner outlier is detected by using the intensity difference between the corner point of the $8 \times 8$ block and its neighbors. At the second stage, the detected corner outlier and its neighbors are replaced with new values calculated by the weighted average method.

Section 2 describes the proposed postprocessing algorithm for blocking effect reduction in JPEG decompressed images. Computer simulations was performed to verify the performance of the proposed algorithm, whose results are presented in section 3 . Finally, conclusions are given in section 4 . 


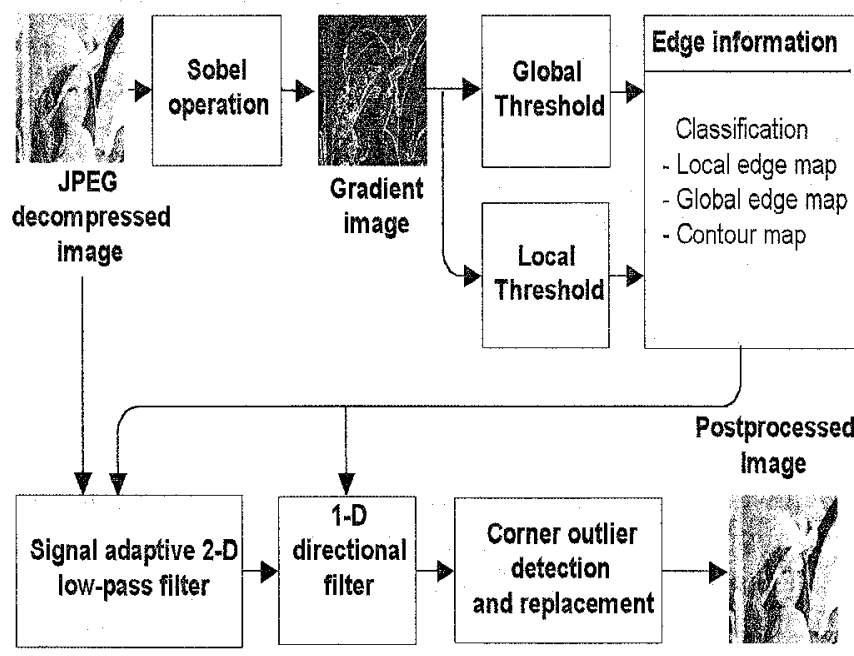

Fig.1. Overall block diagram of the proposed postprocessing algorithm.

\section{PROPOSED ALGORITHM FOR REDUCTION OF BLOCKING EFFECTS}

\subsection{The Property of the JPEG Decompressed Image Gradient}

Figure 1 shows the overall block diagram of the proposed algorithm. The proposed algorithm is based on the edge information, which is the thresholded gradient image. Basically, the idea underlying most edge detection methods is the computation of a derivative, i.e., image differentiation. Gradient operators measure the gradient of the image in a specified direction [6][7]. There is an interesting property of the gradient absolute image computed by the Sobel gradient operator, which is shown in Fig. 2. The gray level of the gradient absolute image has the property that $R=S=2 Q$, where $R$ is a gray level in the gradient absolute image, $\mathrm{S}$ is the slot interval in the histogram of Fig. 2.a, and Q is the quantization factor

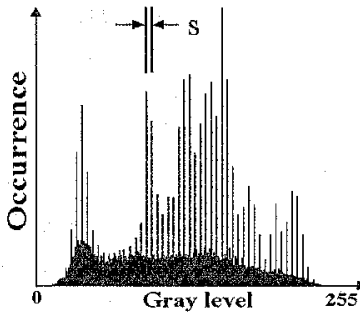

(a)

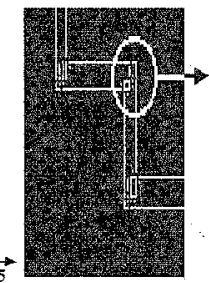

(b)
Fig.2. Property of the JPEG decompressed image:(a) Histogram of the JPEG decompressed image;(b) Magnified view of the grid noise property of the gradient absolute image where each square is a pixel. in the JPEG compression. As shown in Fig. 2.b, all grid noises in the monotone area are less than 6R. For example, when the $Q$ factor is set to 8 , all grid noises in the gradient image are less than 100 . Therefore, if we choose a global threshold value of 100 , the grid noise will not be included in the global edge map.

\subsection{Classification}

The JPEG decompressed image is classified into two areas, such as an edge area and a monotone area, according to the edge map, which is generated by thresholding the gradient absolute image. In general, thresholding is a typical approach to image classification. A global threshold value $T_{g}$, which has a value of 100 in this paper, for the global edge map is chosen in consideration with the $Q$ factor and the histogram characteristics of the gradient image. The performance of the proposed algorithm does not critically depend on the global threshold value.

In addition to the global threshold, local thresholding is performed for the local edge map of each $8 \times 8$ block. The local threshold value $T_{n}$ of the $n$-th $8 \times 8$ block is defined by the characteristics of the pixels within the block. It can be calculated as follows [7],

$$
T_{n}=\left|1-\frac{\sigma_{n}}{m_{n}}\right| * T_{g}
$$

The parameters $m_{n}$ and $\sigma_{n}$ are the mean and the standard deviation of the $n$-th $8 \times 8$ block of the gradient absolute image, respectively. $T_{g}$ is the global threshold value.

If the $n$-th DCT block is homogeneous, the ratio $\sigma_{n} / m_{n}$ tends to be zero with the result that the threshold value $T_{n}$ tends toward $T_{\mathrm{g}}$. If the $n$-th DCT block is complex, the ratio $\sigma_{n} / m_{n}$ is increased, resulting in the threshold value $T_{n}$ being smaller than $T_{g}$. This small $T_{n}$ generates a detailed edge map which is not classified as a global edge by $T_{g}$. Therefore, the local edge map is computed by locally thresholding the $6 \times 6$ pixels within the $8 \times 8$ block excluding boundary pixels in order to preserve the detailed information from blurring and to prevent the grid noise of the block boundary from being detected as an image edge.

\subsection{Signal Adaptive Filter}

A signal adaptive filter is proposed to reduce both the grid noise in the relatively monotone area and the staircase noise along the image edge without any significant loss of the image details. In the first step of 
the signal adaptive filtering, a structure window scans the given global edge map in order to obtain the contour map as shown in Fig. 3. If the origin and the other four points in the structure window are all included in the global edge map, the origin point is excluded from the contour map. After the contour map is obtained from the global edge map, 1-D directional filtering of the JPEG decompressed image is performed for all points on the contour map in order to reduce the staircase noise.

A 1-D directional smoothing filter aligned parallel to the edge is ideally suited for the reduction of staircase noise, which is visible along the image edge. The three coefficients of the 1-D smoothing filter are 1, 4, and 1 .

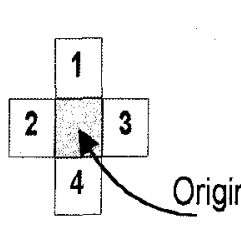

(a)

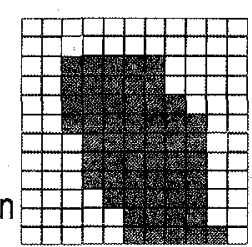

(b)

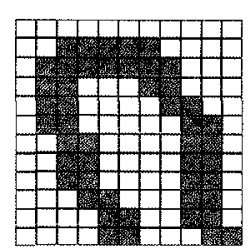

(c)
Fig.3. Contour pixel classification scheme for one-dimensional filtering: (a) Structure window; (b) Global edge map; (c) Contour map.

In the second step, signal adaptive 2-D low-pass filtering is performed. When the central point, point 11 , of the filter kernel in Fig. 4.a is on the global or the local edge map, no 2-D filtering is performed for this point. If any edge point of the global or the local edge map is not included in the $5 \times 5$ filter window, unweighted average filtering is performed by using kernel 1 in Fig. 4.b. If some edge pixels are in the $5 \times 5$ filter window except on the central point, the weighted average filtering is performed by using kernel 2 in Fig. 4.c. In the filter operation using kernel 2 , if any edge point is on the points $12,7,6,5,10,15,16$, or 17 in Fig. 4.a, the weights of the edge pixel and its outer neighbor pixels are set to zero. The weight value is changed according to the position of the edge pixel in the filter window to protect the image details.

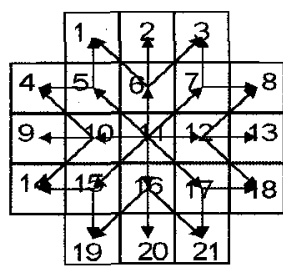

(a)

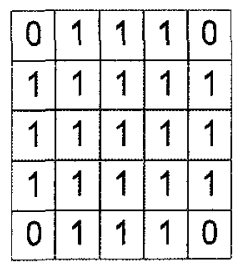

(b)

\begin{tabular}{|l|l|l|l|l|}
\hline 0 & 1 & 1 & 1 & 0 \\
\hline 1 & 2 & 2 & 2 & 1 \\
\hline 1 & 2 & 3 & 2 & 1 \\
\hline 1 & 2 & 2 & 2 & 1 \\
\hline 0 & 1 & 1 & 1 & 0 \\
\hline
\end{tabular}

(c)
Fig.4. Signal adaptive 2-D filter kernels: (a) The coordination of a signal adaptive 2-D LPF kernel; (b) Kernel 1; (c) Kernel 2.

\subsection{Corner Outlier Detection and Replacement}

A corner outlier [10][11] is characterized by a pixel which is either much larger or much smaller than its neighboring pixels in the corner point of the $8 \times 8$ DCT block in the JPEG decompressed image. Each pixel in the corner point is compared with its neighbors in order to detect the corner outlicr. A detected corner outlier and adjacent pixel will be replaced by the weighted average of adjacent corner points with the ratio of $3: 1: 1: 1$. This detection and replacement process is performed in the JPEG decompressed image.

\section{SIMULATION RESULTS}

The proposed postprocessing algorithm was applied to sample images such as Lena, Pepper, and Zelda, all of which were compressed with the standard JPEG algorithm. Resolutions of the images were all $512 \times 512$ pixels with 8-bit gray-levels. Graphical and comparison results are presented for only the Lena image in this paper, since the simulation results for the other images are similar to those of the Lena image.

PSNR of the postprocessed image from the Ramamurthi algorithm and the proposed algorithm are summarized in Fig. 5. The performance of the proposed algorithm is better than that of the Ramamurthi algorithm. The gradient absolute images obtained by the Sobel operation can show the performance of the grid noise reduction by the postprocessing algorithms. The grid noise along the $8 \times 8$ DCT block boundary is most visible in the JPEG decompressed image, whereas the noise is mostly removed in the postprocessed image from the proposed algorithm as shown in Fig.6.

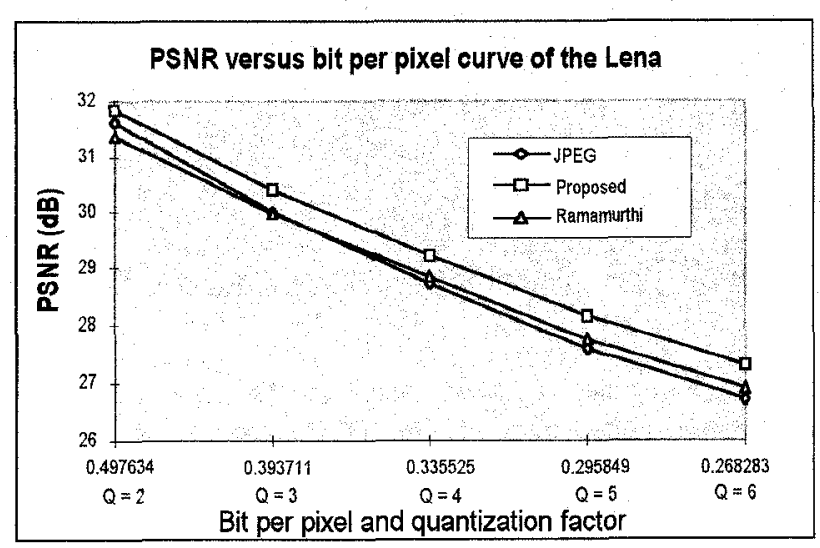

Fig.5. PSNR comparison between the JPEG decompressed image, the postprocessed image by the Ramamurthi algorithm, and the postprocessed image by the proposed algorithm for the Lena image. 


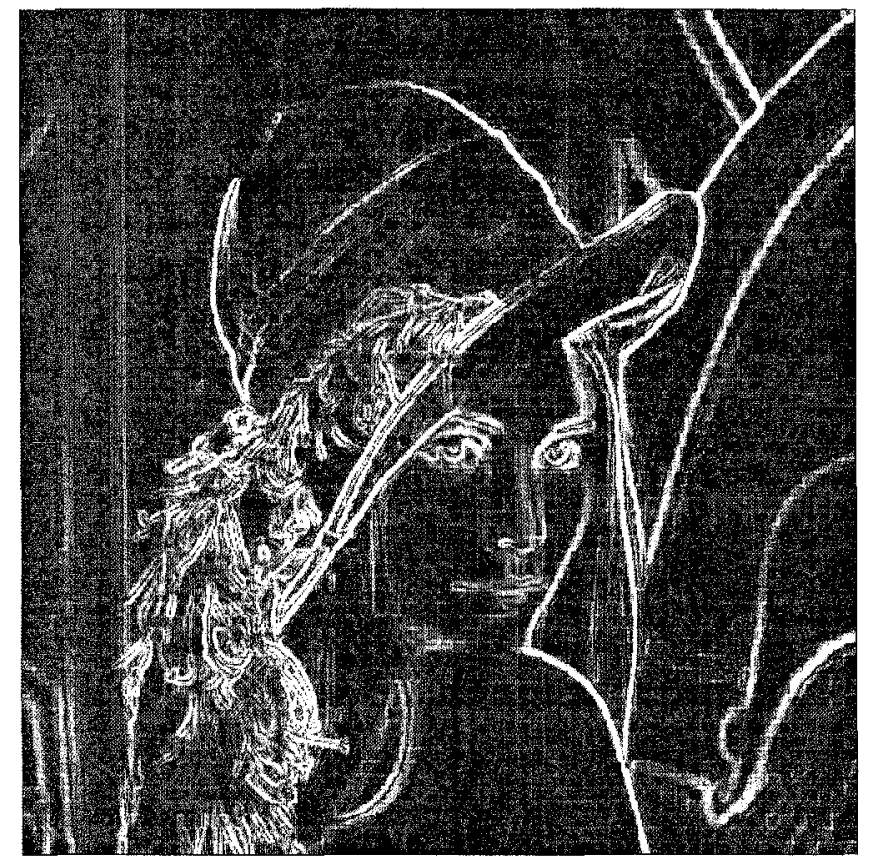

(a)

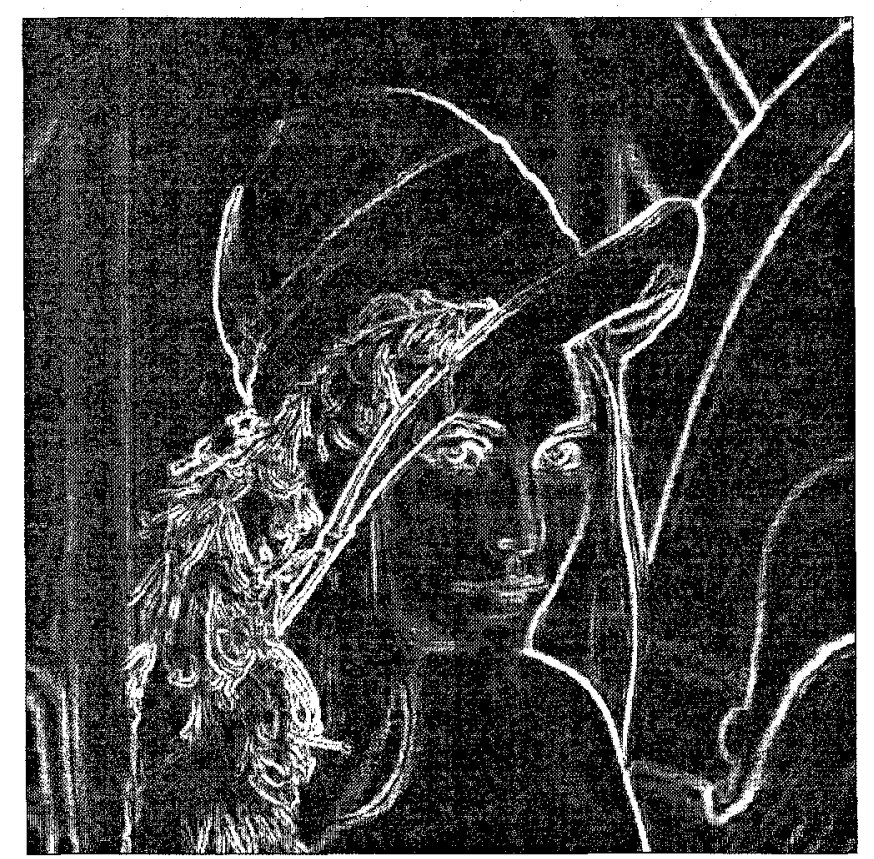

(b)

Fig.6. The gradient absolute image of the Lena image: (a) Gradient absolute image of the JPEG decompressed image, lena.jpg; (b) Gradient absolute image of the postprocessed image by the proposed algorithm.

\section{CONCLUSIONS}

In this paper, a new blocking-effect-reduction algorithm was proposed to improve the quality of the JPEG decompressed image. To reduce the blocking effects without degradation of the image details, the proposed algorithm uses signal adaptive filtering as well as a corner outlier detection and replacement scheme.

The objective performance of the proposed algorithm was measured by the PSNR of the postprocessed image from the proposed algorithm. The measured PSNR showed an increase of $0.2 \sim 1.0 \mathrm{~dB}$ for the three test images of the Lena, the Pepper and the Zelda images at several compression ratios. However, the PSNR does not fully reflect the enhancement of the image quality in the psychovisual viewpoint. From a subjective view, the blocking effects were almost removed in the gradient absolute image made by a Sobel operation. In addition, a visual improvement of the image quality was found in the postprocessed image from the proposed algorithm.

In conclusion, the proposed postprocessing algorithm effectively reduces the blocking effects and well preserves and enhances JPEG images without any increase in the bit rates.

\section{REFERENCES}

[1] W. B. Pennebaker and J. L. Mitchell, JPEG Still Image Data Compression Standard, Van Nostrand Reinhold, New York, 1993.

[2] M. A. Sid-Ahmed, Image Processing, McGRAWHILL, New York, 1994.

[3] B. Ramamurthi and A. Gersho, "Nonlinear Space Variant Postprocessing of Block Coded Images," IEEE Trans. on ASSP, vol. 34, no. 5, pp. 12581267, 1986

[4] B. Zeng and A. N. Venetsanopoulos, "A JPEGbased Interpolative Image Coding Scheme," ICASSP, pp. 393-396, 1993.

[5] W. E. Lynch, A. R. Reibman, and B. Liu, "Post Processing Transform Coded Images using Edges," ICASSP, pp. 2323-2326, 1995.

[6] A. K. Jain, Fundamentals of Digital Image Processing, Prentice-Hall, New Jersey, 1989.

[7] I. Pitas, Digital Image Processing Algorithms, Prentice-Hall, New York, 1993.

[8] W. Lynch, A. R .Reibman, and B. Liu, "Edge Compensated Transform Coding," ICASSP, pp. 105-109, 1994.

[9] S. M. Kay and G. J. Lemay, "Edge Detection Using The Linear Model," IEEE Trans, on ASSP, vol, ASSP-34, no. 5, pp. 1221-1227, 1986.

[10] W. K. Pratt, Digital Image Processing, John Wiley \& Sons, New York, 1991.

[11] X. You and G. Crebbin, "A Robust Adaptive Estimator for Filtering Noise in Images," IEEE Trans. on Image Processing, vol. 4, no. 5, pp. 693699,1995 